\title{
Indications and complications of percutaneous endoscopic gastrostomy with jejunal extension tube
}

\author{
R. C. Rakshit ${ }^{1}$, A. Tawil ${ }^{1}$ and J. Nightingale ${ }^{2}$ \\ ${ }^{1}$ Leicester Royal Infirmary, Infirmary Square, Leicester LE1 5WW and ${ }^{2}$ St Mark's Hospital, Northwick Park, Watford Road,
} Harrow, Middlesex HAl $3 U J$

Percutaneous endscopic gastrostomies with jejunal extension tubes (PEGJ) have been inserted since the early 1980s with variable success $^{(1)}$. This study aimed to review the indications and outcomes of all PEGJ inserted in one teaching hospital from 2002 to 2005 . All the PEGJ were Freka PEG tubes (size 15 French) with jejunal extensions (JE; size 9 French). Long $(300 \mathrm{~cm})$ forceps were used to guide the tip of the JE beyond the duodeno-jejunal (DJ) flexure. Patients were retrospectively identified through the hospital endoscopy database.

Case notes for thirty-six patients (fifteen women) were identified. The mean age was 49.69 (SD 3.61) years. Mean BMI pre-PEGJ was $23 \mathrm{~kg} / \mathrm{m}^{2}$ (range 18-27.5). Fifty-six percent (nineteen of thirty-six) of the cases had haematological indications for PEGJ of which sixteen had hemopoietic stem cell transplants (HSCT) and two cases of acute lymphocytic leukaemia and one with aplastic anaemia who needed nutritional support. The other seventeen included those with serious trauma $(n 3)$, cerebrovascular events $(n$ 6), malnutrition due to tuberculosis $(n 2)$, retroviral disease $(n 1)$, motor neurone disease $(n 1)$, failed naso-jejunal tube feeding $(n 2)$ and lung cancers $(n 2)$.

There was a $91 \%$ (thirty-three of the thirty-six) success rate of inserting the JE beyond the DJ flexure as reported by the endoscopists and X-rays (done within $4 \mathrm{~d}$ post-procedure) confirmed post-pyloric position in $85 \%$ (twenty-three out of twenty-seven) of the patients. Early displacement (within 3 weeks) of the tip of the JE occurred in $36 \%$ (twelve of thirty-three) of the patients. Two-thirds of these were in the haematological group. Fifty percent (eight out of sixteen) of the HSCT patients had early displacement of the tube (usually due to uncontrolled vomiting) and $75 \%$ (twelve out of sixteen) had infections at the entry site. However, at day 60, 40\% (twelve out of thirty) patients still had their PEGJ in place. The insertion site infection rate was $42 \%$ of which $86 \%$ were haematological patients. All cause 30 -d mortality was $17 \%$ (six of the thirty-six). On day 3, 77\% of the patients had more than $75 \%$ of their nutritional requirements met. The mean time between PEGJ insertion and HSCT was 13 (range 1-34) d.

In conclusion, there was a high success rate of initial placement of the JE due to usage of long forceps. Complications were higher in the HSCT patients. Strict guidelines for patients with PEGJ should ensure better outcomes in patients having PEGJ especially those having HSCT.

1. Ponsky JL \& Aszoid A (1984) Am J Gastroenterol 79, 113-116. 